\title{
Face Recognition based on GSVD
}

\author{
Nidhal Khdhair El Abbadi \\ University of Kufa, Education College, \\ Department of Computer Science, Najaf, Iraq
}

\begin{abstract}
The task of face recognition has been actively researched in recent years because of its many applications in various domains. This study suggested new and novel method to matching the input face image with face images in database based on GSVD. Euclidian distance for the norm of two factors resulted from GSVD of each pair of images was determined. When the distance less or equal to specific threshold we decide the two images matched. The accuracy of this proposed algorithm was high comparing with other algorithms and it reached up to $97.21 \%$. The algorithm tested with images from ORL database and Indian face database.
\end{abstract}

\section{Keywords}

Face Matching, Face Recognition, GSVD, Biometrics

\section{INTRODUCTION}

Recognizing a face based attributes is an easy task for a human to perform; it is closely automated and requires little mental effort. A computer, on the other hand, has no innate ability to recognize a face or a facial feature and must be programmed with an algorithm to do so. Generally, there are many types of facial features used in the face recognition [1].

There are two approaches to face recognition. The first one is based on finding the relationship between some of the face features like the eyes, nose and mouth to match the faces. While the second one is template matching using the holistic features of the face images [2].

Fingerprints removed from the crime scene, drop of blood on the floor were used for decades in the forensic as clues to reach the criminals. But with rapid increase with a surveillance camera and mobile devices with built-in cameras, the need emerged to use images in forensic and for that match captured image with very huge of images database.

However, although recent research advances have helped laid the foundations for realizing face-matching scenarios for utilizing this data, face recognition in the forensics area still poses a number of challenges.

Face recognition attract many researcher to developed new algorithms during the past few years.

Zhang et al. (2012) introduced algorithm based on curvelet to catching the edge singularities and two-dimensional principle component analysis (2D PCA) instead of using PCA for face recognition. The input image decomposed to curvelts sub-bands image and then creates a representative feature set by applying 2D-PCA [3]

Wan et al. (2010) proposed algorithm called the Fuzzy Local Discriminate Embedding (FLDE), in this algorithm the researcher try to correct the information distribution by reducing the outer effects by using the Fuzzy K-Nearest Neighbor (FKNN). In this study the auther calculated the a membership degree matrix by using FKNN, then the membership degree is incorporated into the definition of the Laplacian scatter matrix to obtain the fuzzy Laplacian scatter matrix. The optimal projections of FLDE can be obtained by solving a generalized Eigen function [4].

Kumar M.S.S. et al. (2011) introduced face recognition based on partitioning image into smaller sub-divisions from a predefined neighborhood and they were ultimately fused to acquire many sets of features. Since a few of the normal facial features of an individual do not differ even when the pose and illumination may differ, the proposed method manages these variations [5].

\subsection{Generalized Singular Value Decompositions (GSVD)}

The Singular Value Decomposition (SVD) of a matrix is one of the most important tools in mathematics. It has a long history dating back to the work of Sylvester, Autonne, Eckart and Young and many others. There are now a number of extensions of the SVD involving factorization of more than one matrix, that are collectively termed generalized singular value decompositions: i.e., GSVDs.

In linear algebra, the Generalized Singular Value Decomposition (GSVD) is the name of two different techniques based on the singular value decomposition. The two versions differ because one version decomposes two (or more) matrices (much like higher order PCA) and the other version uses a set of constraints imposed on the left and right singular vectors.

The SVD and GSVD have diverse applications involving areas such as signal processing, numerical computation and statistics. In particular, Canonical Correlation Analysis (CCA) in statistics is closely related to the SVD. The SVD and GSVD can also be applied to analyze and to solve leastsquares problems in numerical analysis.

Many algorithms to compute the GSVD have been proposed, in this study we used the Matlab to determine the results, Note that Matlab has a built-in GSVD.

$[\mathrm{U}, \mathrm{V}, \mathrm{X}, \mathrm{C}, \mathrm{S}]=\operatorname{gsvd}(\mathrm{A}, \mathrm{B})$ returns five matrices, $\mathrm{U}$ and $\mathrm{V}$ are both unitary matrices, and two diagonal matrices with nonnegative values $(\mathrm{C}$, and $\mathrm{S})$, and finally one square matrix $\mathrm{X}$. from these matrices we can rebuild (A, and B) as follow:

$\mathrm{A}=\mathrm{U}^{*} \mathrm{C}^{*} \mathrm{X}^{\prime}$

$\mathrm{B}=\mathrm{V}^{*} \mathrm{~S} * \mathrm{X}^{\prime}$

$\mathrm{C}^{\prime} * \mathrm{C}+\mathrm{S}^{\prime} * \mathrm{~S}=\mathrm{I}$

Note that the number of coloums for A and B must be the same, while there is no restriction for numbers of rows. If $A$ is $m-b y-p$ and $B$ is $n-b y-p$, then $U$ is $m-b y-m, V$ is $n-b y-n$ and $X$ is $p-b y-q$ where $\mathrm{q}=\min (\mathrm{m}+\mathrm{n}, \mathrm{p})$.

When $\mathrm{B}$ is square and nonsingular, the generalized singular values, gsvd $(\mathrm{A}, \mathrm{B})$, are equal to the ordinary singular values, svd $(\mathrm{A} / \mathrm{B})$, but they are sorted in the opposite order. Their reciprocals are gsvd $(B, A)$.

Note, however, that when null (A) and null (B) do overlap, the nonzero elements of $\mathrm{C}$ and $\mathrm{S}$ are not uniquely determined. 


\section{MATERIALS AND METHODS}

There are many problems accompany with face matching, starting from the different expression for same face in different images, passing to different illumination and noise due to capturing the images with different times and different environments and ended to images with faces with different directions. All these issues and other add further difficulties to recognize face and matching specific face with the face images in database.

This study suggested new and novel method to matching the input face image with face images in database based on GSVD. The face images used in this study from ORL database and Indian face database were shot at different times and with different lighting conditions. The input image will be compare with all database images by determine the GSVD for the input image with each image in database and determine which image in database is matching with input image or more closes to input image according to suggested threshold. In this method the color image will be either convert to gray image or select one channel of image colors (red, green, or blue), both of them give the same results. The images resized to reduce the process image size which increases the program performance.

Suppose the input image A required to match it with images in the database of faces images. $\mathrm{Bi}$ is one of the database images where $(i=1,2,3, \ldots$ No. of images in database). To find whether image $\mathrm{Bi}$ matching to image $\mathrm{A}$ we determine the GSVD as in the relation (1):

$$
[\mathrm{U}, \mathrm{V}, \mathrm{X}, \mathrm{C}, \mathrm{S}]=\mathrm{GSVD}(\mathrm{A}, \mathrm{Bi})
$$

From the result of relation (1) we determine the norm for each of $(\mathrm{C}$ and $\mathrm{S})$ and then determine the Euclidian distance between (norm $\mathrm{C}$ and norm $\mathrm{S}$ ):

\section{GSVD Distance $=$ abs $(\mathrm{C}-\mathrm{S})$}

The GSVD Distance will be compare with threshold suggested for this purpose. When the GSVD distance value less or equal to threshold this mean the image is highly similar to the input image.

\section{RESULTS}

This proposed algorithm tested with many images and in different cases as follow:

The algorithm tested with many images for the same face with different expression as shown in Fig. 1, the GSVD distance calculated for each pair of images in Fig. 1. And the result was $100 \%$ matching.

The algorithm tested with different faces images as in Fig. 2 and the result was matching up to $94 \%$. In this case we have to mention that the race of people in the images will highly affect on the matching results.

We tested the proposed algorithm with different threshold to find the best threshold can used for matching. The experiment results showed that the threshold range between $0.15-0.2$ is the best which give performance up to 97.21 true matching (true matching mean the two compared image either true matched or rue unmatched), as showed in Fig. 3.

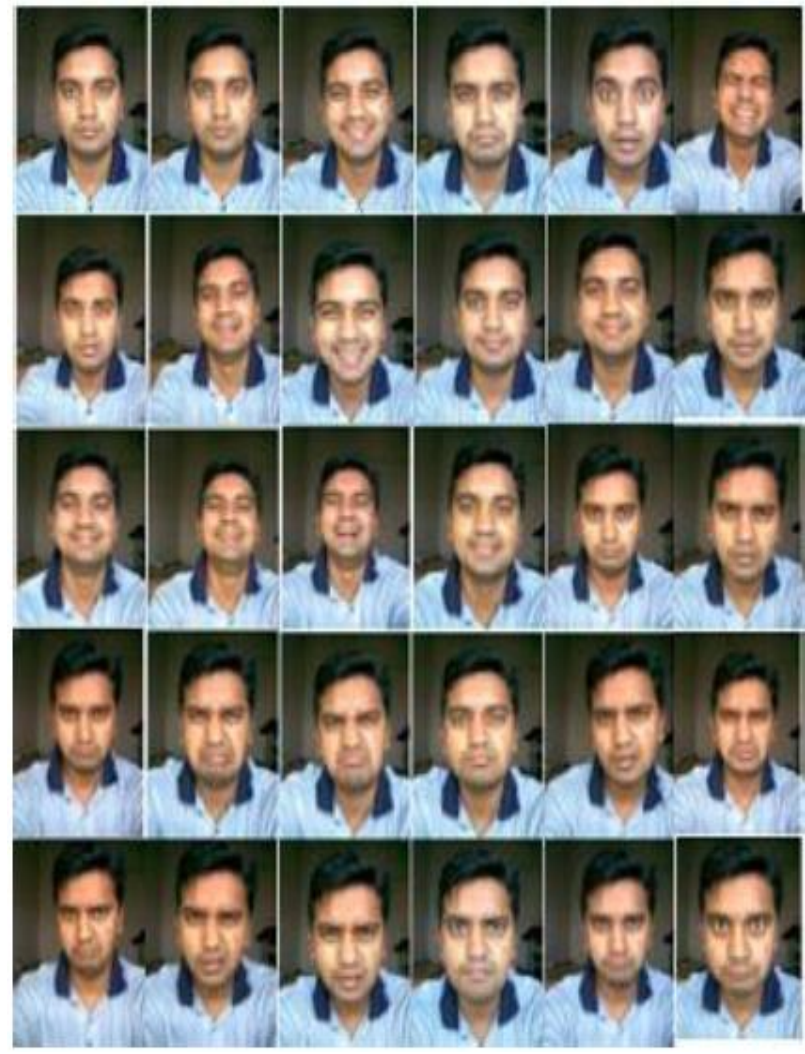

Fig. 1. Face images with different expression

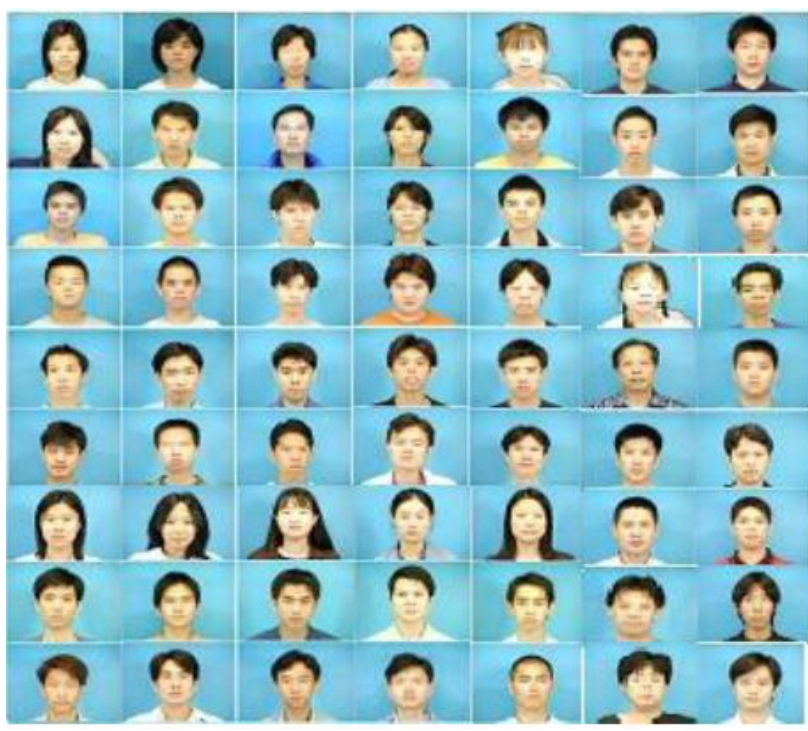

Fig. 2. Different faces images

It was seen that the GSVD Distance will be vary according to image size used in the program (image when resized in program prior to process) as shown in Fig. 4. Also the execution time varies with variation of image size as shown in Fig. 5. 


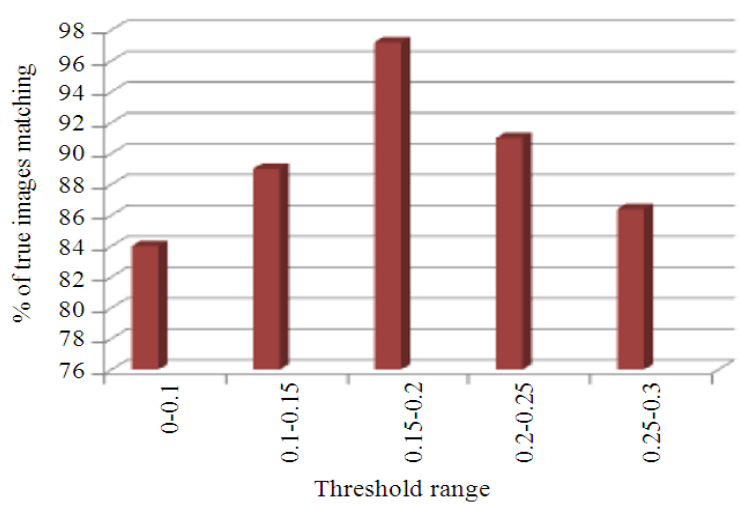

Fig. 3. Comparing image matching with different thresholds

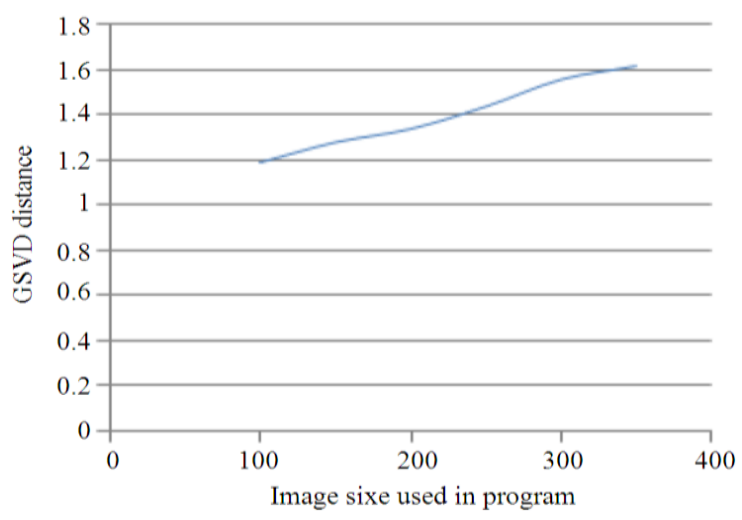

Fig. 4. Comparing image size used in program with GSVD distance

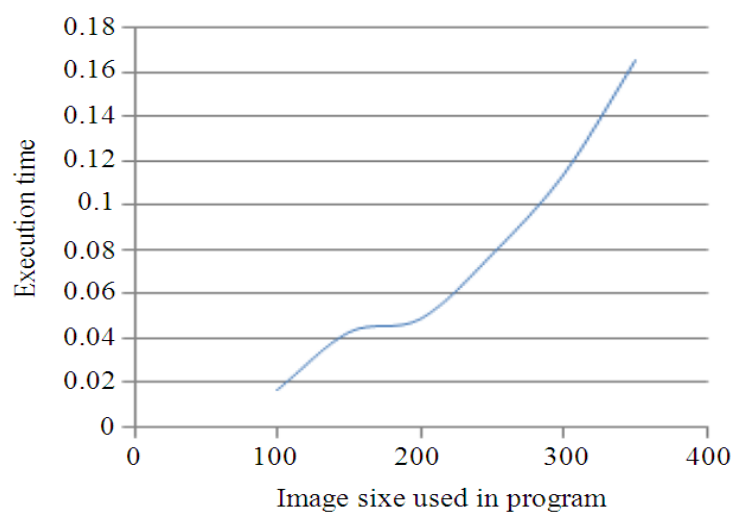

Fig. 5. Comparing image size used in program with program execution time

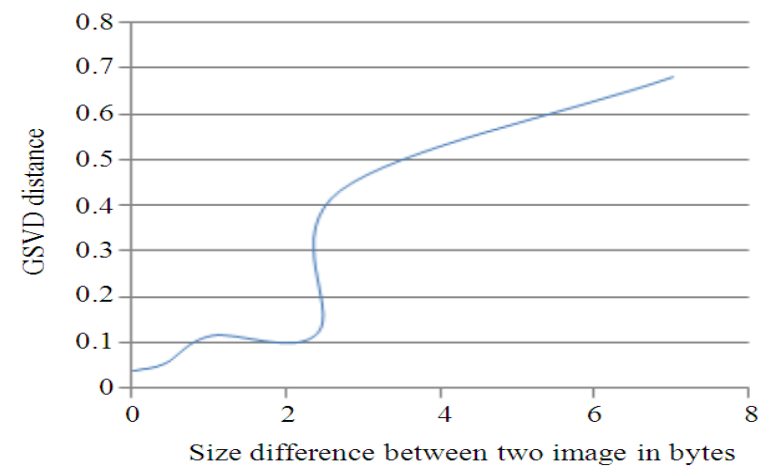

Fig. 6. Shows how the differences in the input image size affected on the GSVD Distance

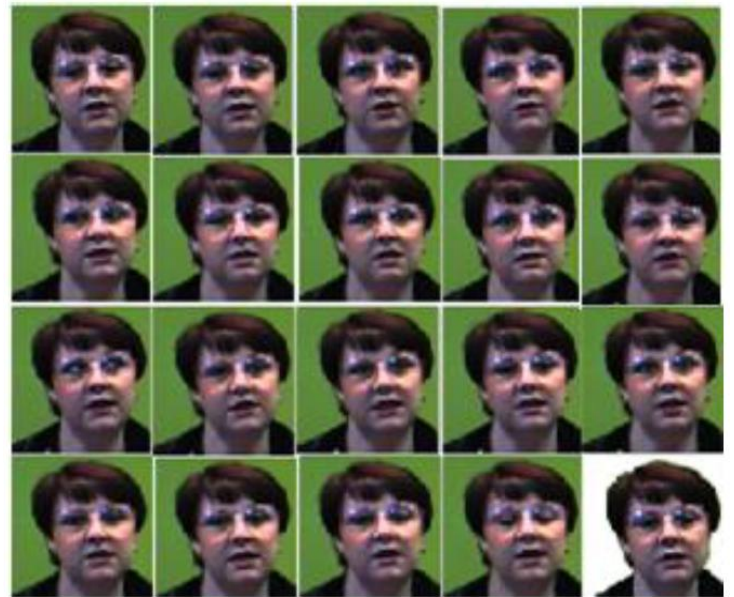

Fig. 7. Images for test the matching when changing background

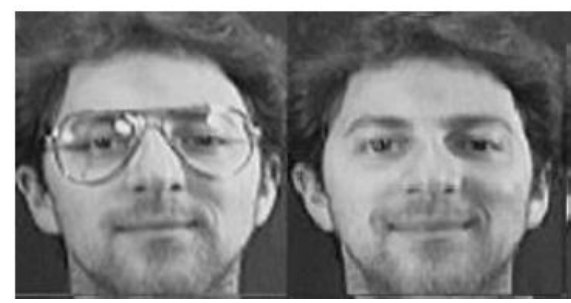

Fig. 8. Same face with and without glasses

Best result can get when the two images originally with the same size, increasing the size difference between the two input images will increase the GSVD Distance. Figure 6 Shows how the GSVD Distance affected with increasing the difference in the image size.

Table 1. Comparing proposed algorithm with other algorithms

\begin{tabular}{llc}
\hline & \multicolumn{2}{l}{ Accuracy } \\
Methods & $\%$ & (SECS) \\
\hline Principal Components Analysis (PCA) & 92.00 & 25.77 \\
PCA+SVD & 94.00 & 17.89 \\
Fuzzy Local Discriminant (FLD) & 94.00 & 36.98 \\
FLD+SVD & 86.25 & 29.33 \\
Fuzzy Local Discriminant & 93.60 & \\
Embedding (FLDE) & & \\
Linear Discriminate Analysis (LDE) & 88.21 & \\
Eigenface & 91.33 & \\
Wavelet + PCA & 94.47 & \\
Curvelet based on 2D-PCA & 94.29 & \\
Proposed algorithm & 97.21 & 32.00 \\
\hline
\end{tabular}

Image background color will affect the image matching; best result can achieve when both input images have the same background color. In Fig. 7 the image with white background did not match any of the other images with green background, while all the other images matches each other according to suggested threshold.

Wearing glasses can make some difficulties to matching the image as shown in Fig. 8. The faces did not match in this figure although it is for the same face. 
This algorithm compared with other face matching algorithms and gives very good result as shown in Table 1 [3].

\section{CONCLUSION}

In this study we introduced new method for matching faces based on GSVD. The results were promised and better than many other algorithms used for face matching.

There are many factors affected on face matching, then if we take care for these factors we will enhance the image matching, some of them the distance from the camera should be fixed if the distance differ from one image to other this will yield different GSVD distance. Other factor affect the matching is the glasses, if the same two images compared one the face with glasses and other without glasses the performance of matching will be degraded. Background and face direction should be noticed. The proposed method took relatively long time due to compare the input image with all the database images, it need about $0.1 \mathrm{sec}$ for each pair of image. This time can be reduced if the database images classified according to some features like separating male images from female images. Face down, illumination, human race and the clothes all affect the matching.

I suggest using same image size which will increase the performance.

For future works I suggest to segment the face from background and then determine the GSVD distance. Also I suggest comparing the threshold for each human race and determining the best distance from camera.

\section{REFERENCES}

[1] Sakthivel, S. and M. Rajaram, 2011. Improving the performance of machine learning based multi attribute face recognition algorithm using wavelet based image decomposition technique. J. Comput. Sci., 7: 366-373. DOI: $10.3844 / j \operatorname{cssp} .2011 .366 .373$.

[2] Rani, J.S., 2012. Face recognition using hybrid approach. Int. J. Image Graphics, 12: 1-27. DOI: $10.1142 / \mathrm{S} 0219467812500052$.

[3] Zhang, Y., B. Yu and H.M. Gu, 2012. Face recognition using curvelet based two dimensional principle component analysis. Int. J. Patt. Recogn. Artif. Intell., 26: 1-13. DOI: $10.1142 / \mathrm{S} 0218001412560095$.

[4] Wan, M., G. Yang, Z. Lai and Z. Jin, 2010. Feature extraction based on fuzzy local discriminate embedding with applications to face recognition. IET Computer Vision, 5: 301. DOI: 10.1049/iet-cvi.2011.0028.

[5] Kumar, M.S.S., R. Swami and M. Karuppiah, 2011. An improved face recognition technique based on modular LPCA approach. J. Comput. Sci., 7: 1900-1907. DOI: 10.3844/jcssp.2011.1900.1907.

[6] Noushath, S., A. Rao and G.H. Kumar, 2007. SVD based algorithms for robust face and object recognition in robot vision applications. Proceedings of the 24th International Symposium on automation and Robotics in Construction, (ISA 07), pp: 473-477. 